\title{
Performance Comparison of Different Pilot Aided Channel Estimation Techniques for OFDM IEEE 802.11e (Mobile Wimax) Systems
}

\author{
Rajeshwar Singh \\ Lovely Professional University \\ Punjab,INDIA
}

\author{
Gurpreet Singh Saini \\ Lovely Professional University \\ Asst.Professor
}

\begin{abstract}
Orthogonal frequency-division multiplexing (OFDM) modulation is a promising technique for achieving the high bit rates required for a wireless multimedia service. In this paper we present different channel estimation algorithms for OFDM mobile communication systems based on the use of pilot subcarriers. Specifically we present LS (Least Square), MMSE (Minimum Mean Square Error) and LMS iterative channel estimation techniques to show the performance comparison of channel estimation techniques supported by $n$ IFFT-based time channel estimation. The channel estimation is based on the principle of pilotsymbol aided channel estimation (PACE), and it has been implemented using block-type pilot subcarrier arrangements. The low complexity of LMS algorithm, has a higher efficiency than conventional methods (without channel estimation, LS\& LMMSE) and it can work in lower amount of SNRs. We have compared the performances of all schemes by measuring bit error rate with 16QAM as modulation scheme, and multi-path Rayleigh fading channel as channel model. Simulation results validate that the LMS Iterative channel estimation scheme can achieve tremendous performance as the existing channel estimation methods.
\end{abstract}

Keyword: Channel estimation (CE), OFDM (Orthogonal Frequency Division Multiplexing), QAM (Quadrature Amplitude Modulation).

\section{INTRODUCTION}

Future generation wireless communication systems demand multiple access schemes with high data transmission capabilities. One such potential multiple access scheme is the Orthogonal Frequency Division Multiple Access. OFDM has received growing interest recently for its use in wireless and wireline communication systems[1] [2]because of its high rate transmission capability with high bandwidth efficiency and robustness to multipath fading and delay. Since OFDM employs overlapped carriers, its spectrum efficiency is also very good. In most of applications, for retaining the orthogonality of subcarriers and overcome intersymbol interference (ISI), a cyclic prefix (CP) is inserted instead of simply inserting guard interval. If the maximum delay of the Multipath channel does not exceed the CP length, the OFDM system would be ISI free by removing the guarding interval. OFDM based systems are strong candidates for air interface of future fourth generation mobile wireless systems which provides high data rate and mobility.
In order to reveal the coherent of received signals, channel coefficients must be estimated by a process so called Channel Estimation. The channel estimation can be performed by inserting pilot sub carriers at the transmitter side and channel coefficients that belong to the pilot subcarriers are estimated using the LS (Least Square) method. Channel estimates are derived [4] from scattered pilots. The pilot carriers in each OFDM symbol contain data known a priori to the receiver. Samples of the channel transfer function can be generated by removing the pilot carrier modulation. Interpolation of these samples can generate the channel estimates for the remaining carriers in the symbol.

As for channel estimation, there have been a variety of algorithms with different optimization criteria and levels of numerical complexity. These channel estimation techniques are based on the type of pilot insertion at the transmitter side. There are basically two types of pilot insertion are used in OFDM systems as shown in Figure 2.The First one is Block Type pilot insertion, has been developed under the assumption of slow fading channel and the second is comb type pilot insertion, has been introduced to satisfy the need for equalizing when the channel changes even in one OFDM block. The estimation of the channel for the block-type pilot arrangement can be based on Least Square (LS) or Minimum Mean-Square (MMSE). The LS channel estimation and LMMSE channel estimation can both be applied to pilot subcarriers assisted channel estimation for OFDM systems. The LS channel estimation is the better approach [3] from the implementation point of view but it suffers the high mean square error (MSE) in regions of low SNR. The LMMSE channel estimation employs the channel statistics to minimize the MSE and yields better performance than the LS channel estimation in regions of low SNR. The major drawbacks of the LMMSE channel estimation are the high complexity of computation and the difficulty of obtaining the channel statistics. Therefore to compensate with complexity and to improve the performance of BER, different iterative channel estimation techniques are available such as EM, RLS and LMS. Among them LMS technique is used due to its less complexity and its acceptable performance. The idea is to feed back information from [5] the output of the channel decoder to the estimation stage. The estimator can improve its performance because it gets not only the information from the pilots but also reliability information of the coded bits. In this paper we demonstrate the performance of different channel estimation techniques i.e. LS,LMMSE and LMS iterative for OFDM based systems.. In this paper, our aim is to compare the performance of all of the above schemes by applying 16QAM (16 Quadrature Amplitude 
Modulation).We evaluate the estimation accuracy of the estimation methods and bit error rate performance. The system with LMS technique provide better results for bit error rate as compared to the LMMSE and LS channel estimators. The LMMSE estimator has good performance but high complexity. The LS estimator has low complexity but its performance is not as good as that of MMSE estimator.LMS technique has less complexity and provide aceeptable BER performance as compared to the other techniques.

\section{SYSTEM DESCRIPTION}

\subsection{SYSTEM MODEL}

In this section we will examine the transmitter and receiver structure of OFDM systems including the pilot distribution. Consider an OFDM system with $N$ subcarriers which employs the quadrature Amplitude Modulation (QAM) modulation. as illustrated in Figure 1.The vector of data bits is first encoded with an error correcting code such as convolutional code to obtain the vector of coded data bits. This vector is mapped accordingly to the constellations and vector of data symbols is obtained. After the guard band is inserted, an N-point Inverse discrete fourier transform (IDFTn) block transforms the data sequence into time domain. Following the IDFT block, a cyclic extension of time length $\mathrm{Tg}$ choosen to be larger than the expected delay spread is inserted to avoid Inter Symbol Interference(ISI) [7-10].The Digital to Analog Converter (D/A) contains low pass filters.. The channel is modeled as an impulse response $\mathrm{h}(\mathrm{t})$ followed by the complex additive white Gaussian Noise $\omega(\mathrm{t})$.

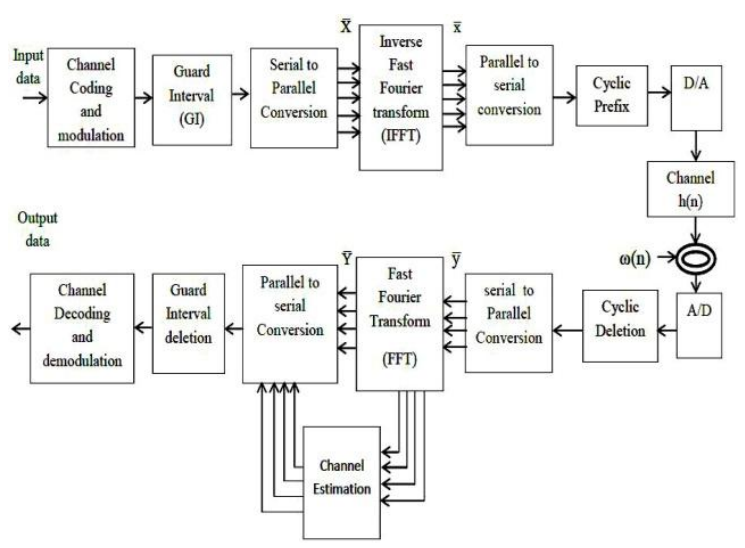

Where,

$\overline{\mathrm{x}}=$ OFDM symbol $\quad \omega=$ Additive White Gaussian

Noise

$\mathrm{h}=$ Channel transfer function $\overline{\mathrm{Y}}=$ Received signal

Figure 1 A digital implementation of OFDM baseband

\section{system}

In many wireless channels, there may be more than one path from a transmitter to a receiver. The incoming radio waves arrive from different directions with different propagation delays. The time delays and attenuation [6] factors of the different paths are generally time-varying in mobile communication and the complex baseband representation of a channel impulse response (CIR) can be described by:

$$
h(t, \tau)=\sum_{m=1}^{M} \alpha_{m}(t) \delta_{D}\left(\tau-\tau_{m}(t)\right),
$$

where $\tau_{m}(\mathrm{t})$ and $\alpha_{m}(t)$ are the delay and the complex amplitude of the $m^{\text {th }}$ path, respectively.

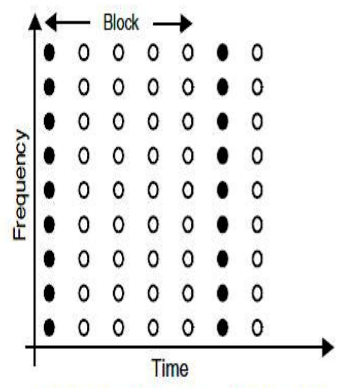

Block-type pilot channel estimation

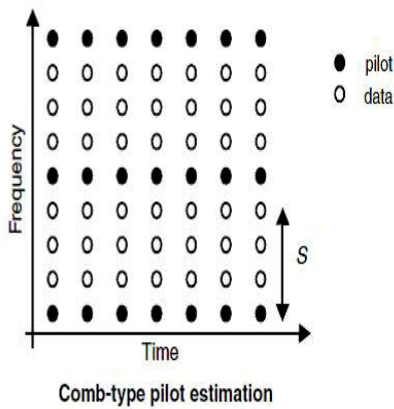

Comb-type pilot estimation
Figure 2 Types of Pilot insertion in OFDM systems

The transmitted symbols are denoted by $\bar{x}=[x(n)]=[x 1(n), x 2(n), x 3(n) \ldots \ldots . x k(n)]$ and the received symbols are denoted by $\overline{\mathrm{Y}}=[\mathrm{Y}(\mathrm{n})]=[\mathrm{Y} 1(\mathrm{n}), \mathrm{Y} 2(\mathrm{n}), \mathrm{Y} 3(\mathrm{n}) \ldots \ldots \ldots \ldots \mathrm{Yk}(\mathrm{n})]$. The transmitted symbols are fed to Inverse Fast Fourier Transform to produce OFDM signal and a guard interval is inserted, which is a cyclic extension of the IDFT output sequence, in order to eliminate the inter-symbol interference (ISI).

Then OFDM frame is transmitted [11] through the time varying frequency selective channels. This channel is described using baseband equivalent impulse response as $\overline{\mathrm{h}}=[\mathrm{h}(\mathrm{n})]=[\mathrm{h} 1(\mathrm{n}), \mathrm{h} 2(\mathrm{n}), \mathrm{h} 3(\mathrm{n}) \ldots \ldots . \mathrm{hl}(\mathrm{n})]$ where $\mathrm{l}$ is the length of channel. We assume $\bar{n}=[\omega(n)]=[\omega 1(n), \omega 2(n)$, $\omega 3(\mathrm{n}) \ldots \ldots \omega \mathrm{c}(\mathrm{n})]$ as additive white Gaussian noise added over channel.

After removing the Cyclic prefix and applying FFT, the received signal is obtained as,

$$
\bar{Y}=\operatorname{DFT}\left(\operatorname{IDFT}\left(\bar{X}^{*} \bar{h}+\bar{n}\right)(1)\right.
$$

Define the input matrix:

$$
\mathrm{F}=\left[\begin{array}{ccc}
W_{N}^{00} & \cdots & W_{N}^{0(N-1)} \\
\vdots & \ddots & \vdots \\
W_{N}^{(N-1) 0} & \cdots & W_{N}^{(N-1)(N-1)}
\end{array}\right]
$$

Here,

$W_{N}^{n, k}=\frac{1}{\sqrt{N}} e^{-j 2 \pi n k / N}$ is called twiddle factor.

Also define $\bar{H}=D F \operatorname{Tn}(\bar{h})=F \bar{h}$ and $\bar{N}=F \bar{n}$

As a matter of convenience we write (1) in matrix notation

$$
\bar{Y}=X \bar{H}+\bar{N}
$$

In the channel estimation unit, the estimate of $\bar{H}$ is obtained with the channel information given by $\bar{H}$, channel equalization is performed by $X=\bar{Y} / \bar{H}$. Finally $X$ is demodulated and original signal is restored. 


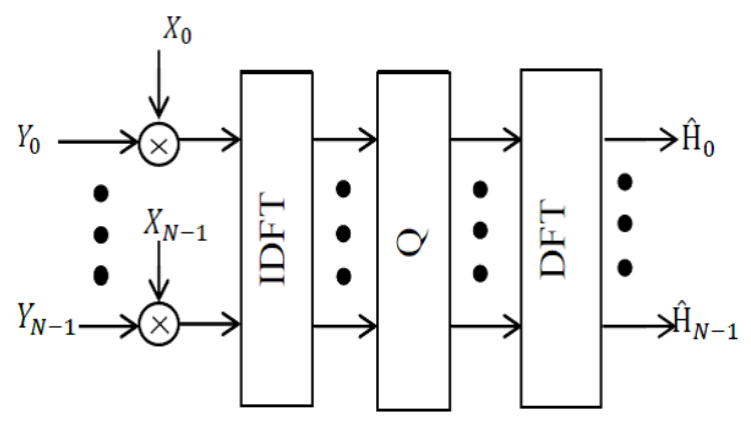

Figure 3: General Estimator Structure

\subsection{PILOT AIDED CHANNEL ESTIMATION}

In pilot aided channel estimation scheme, pilot symbols are added at the transmitter side in which all subcarriers are used as pilots .If we assume that channel is constant then there will no channel estimation error since the pilots are sent at all carriers. The estimation can be performed by two methods. The first one is LS(Least Square) and the second one is MMSE(Minimum Mean Square Error).

\subsubsection{LS Estimator:}

The first step in the channel estimation process is to remove the modulation of the pilot symbols. Thus, an initial estimate of the CTF (Channel Transfer Function) at pilot positions is obtained. The estimate of [12]channel transfer function is obtained by using LS estimator. The LS estimator for the cyclic impulse response $h$ minimizes $(Y-X F h)^{H}(Y-X F h)$ and generates:

Where,

$$
\hat{\mathrm{H}}_{L S}=\mathrm{F} Q_{L S} F^{H} X^{H} Y(3)
$$

$Q_{L S}=\left(F^{\mu} X^{\mu} X F\right)^{-1}$

Note that $\hat{\mathrm{H}}_{L S}$ also corresponds to the estimator structure in Fig. 3. Since (3) reduces to:

$\hat{\mathrm{H}}_{L S}=X^{-1} Y$

The LS estimator is equivalent to what is also referred to as the zero-forcing estimator. Where $(\cdot)^{n}$ means the conjugate transpose operation Without using any knowledge of the statistics of the channels, the LS estimators are calculated with very low complexity, but they suffer from a high mean-square error. When the channel is slow fading, the channel estimation inside the block can be updated using the decision feedback equalizer at each sub-carrier.

\subsubsection{MMSE Estimator}

MMSE Estimator makes full use of the correlation of the channel frequency response at different times and frequencies. In particular, for mobile wireless channels, the correlation of the channel frequency response at different times and frequencies can be separated into the multiplication of the time and frequency domain correlation functions. Hence, MMSE channel estimator can be a frequency-domain filter using the fast Fourier transform (FFT), followed by timedomain filters. Since the channel statistics, which depend on the particular environment, are usually unknown, we present a robust estimator, that is, an estimator that is not sensitive to the channel statistics. If the time domain channel vector $h$ is Gaussian and uncorrelated with the channel noise $\omega$, the frequency domain MMSE [12-13] estimate of $h$ is given by:

$$
\hat{\mathrm{H}}_{M M S E}=F R h Y R_{y y}{ }^{-1}
$$

Where,

$$
\begin{gathered}
R_{H H}=E\left\{\bar{H} \bar{H}^{H}\right\}=E\left\{(F \bar{h})(F \bar{h})^{H}\right\}=F R_{h h} F^{H} \\
R_{h y}=E\left\{\bar{h} \overline{Y^{H}}\right\}=E\left\{\bar{h}(X F \bar{h}+\bar{N})^{H}\right\}=R_{h h} F^{H} X^{H} \\
R_{Y Y}=E\left\{\bar{Y} \overline{Y^{H}}\right\}=X F R_{h h} F^{H} X^{H}+\sigma^{2} I_{N}
\end{gathered}
$$

$R_{h y}$ is the cross covariance matrix between $h$ and $y$ and $R_{Y Y}$ is the auto covariance matrix of $Y . R_{H H}$ is the autocovariance matrix of $H$ and $\sigma^{2}$ represents the noise variance $E\left\{|W(k)|^{2}\right\}$.

These two quantities $R_{h y}$ abd $R_{Y Y}$ are assumed to be known. Since the columns in $\mathrm{F}$ are orthonormal, $\hat{\mathrm{H}}_{M M S E}$ generates the frequency-domain MMSE estimate $\hat{\mathrm{H}}_{M M S E}$ by:

$$
\begin{aligned}
& \hat{\mathrm{H}}_{M M S E}=F Q_{M M S E} \quad F^{H} X^{H} Y \\
& \text { (4) }
\end{aligned}
$$

where $Q_{M M S E}$ can be shown to be:

$$
\begin{aligned}
& Q_{M M S E}=R_{h h}\left[\begin{array}{lll}
( & F^{H} & \left.X^{H} X F\right)^{-1} \sigma n^{2}+R_{h h}
\end{array}\right]^{-1}\left(F^{H}\right. \\
& \left.X^{H} X F\right)^{-1}
\end{aligned}
$$

This MMSE channel estimator (4) has the form shown in Fig. 3.If $h$ is not Gaussian, $\hat{\mathrm{H}}_{M M S E}$ is not necessarily a minimum mean-square error estimator. It is however the best linear estimator in the mean-square error sense.The MMSE estimator yields much better performance than LS estimators especially under the low SNR scenarios.

A major drawback of the MMSE estimator is its high computational complexity, especially if matrix inversions are needed each time the data in $X$ changes.

\subsubsection{LMS ITERATIVE CHANNEL ESTIMATION}

In this section, we propose a low-complexity ICE algorithm with improved error performance which employs the well known LMS algorithm.

The equspaced Pilots are used to obtain estimates of the channel at the receiver side. In this paper we use block type pilot insertion in which pilots are inserted in time domain and at the beginning of a block, we apply a LS (Least Square) estimator to achieve the initial channel estimation, and then at every time interval, the new channel estimation is obtained by performing the LMS (least mean square) algorithm. In LMS technique we provide iterations to the estimated channel. The channel which was estimated in each iteration would be used for next iteration. This estimation in each iteration can be used as side information and feed back to system to achieve better result for next iteration.[14-15] 


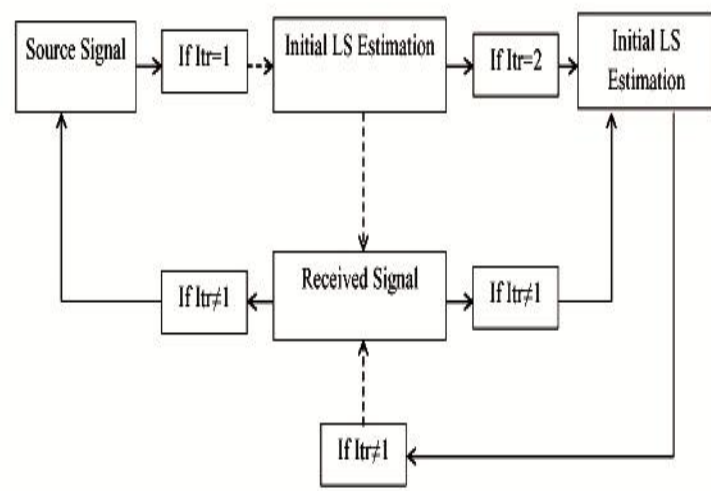

Figure 4 :Implementing LMS Iterative alogirithm

It is given by:

$$
\hat{\mathrm{H}}_{n}=\hat{\mathrm{H}}_{n}-1-h \mu * X *
$$

Where,

- $\mathrm{n}=$ The iteration state

- $\mathrm{e}=$ The signal error

- $\mu=$ A coefficient between $0-1$

As illustrated in figure 4.LMS algorithm is applied to receiver and the channel which was estimated in each iteration would be used for next iteration additionally the output signal is fed to source signal for next channel estimation. Another important factor in channel estimation through this method is $\mu$ which influences on estimation and should be precisely choosen. The performance of LMS algorithm can be closely to LS algorithm by careful choice of $\mu$.

\subsection{Estimator Complexity}

The complexity of LMS iterative estimation is less than the conventional estimators. Notice that LS estimator has much lower complexity than MMSE estimator.

It should be noted that the MMSE estimators have been derived under the assumption of known channel correlation and noise variance. In practice these quantities, $R_{h h}$ and $\sigma^{2}$ are either taken fixed or estimated, possibly in an adaptive way. This will increase the estimator complexity and reduce the performance slightly.

Whereas in LMS iterative channel estimation, estimation in each iteration can be used as side information and feed back to system to achieve better result for next iteration. Thus this technique reduces the complexity and improves the performance.

\section{SIMULATION AND EVALUATION}

In this section we demonstrate the performance of proposed iterative channel estimation method for OFDM systems. In the simulation we consider an OFDM system operating with bandwidth of $8.75 \mathrm{MHz}$,Length of FFT as 1024 and Guard Interval of 1/8.Total number of data bits are inserted uniformly at even and odd positions. System parameters used in the simulation are indicated in Table 1.
Table 1.OFDM system parameters

\begin{tabular}{|l|l|}
\hline Parameters & Values \\
\hline System bandwidth & $8.75 \mathrm{MHz}$ \\
\hline Sampling Frequency & $10 \mathrm{KHz}$ \\
\hline Number of subcarriers & 1024 \\
\hline Cyclic prefix & $1 / 8$ \\
\hline Modulation & 16 QAM \\
\hline Useful symbol Time & $\mathbf{0 . 1 0 2 4 m s}$ \\
\hline OFDM Symbol Time & $\mathbf{0 . 1 1 5 2 m s}$ \\
\hline Guard Time & $\mathbf{0 . 0 1 2 8 m s = 1 2 . 8 u s}$ \\
\hline
\end{tabular}

In the simulation we present 16 QAM modulation scheme. Total number of data bits taken are 1024 and 3456 OFDM symbols. The Flow Chart of the whole simulation process is as shown in the figure 5:

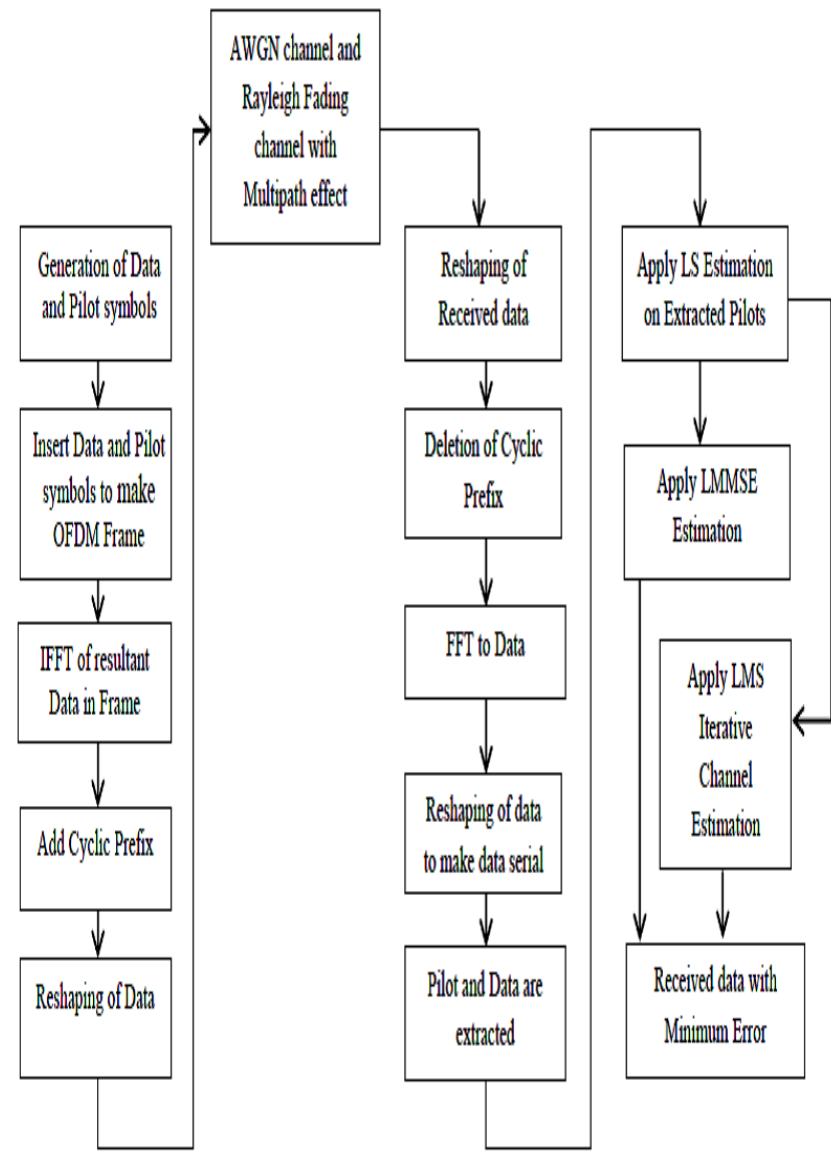

Figure 5: Flow Chart of Simulation Process

The generation of modulated OFDM data symbols is illustrated in figure 6 : 


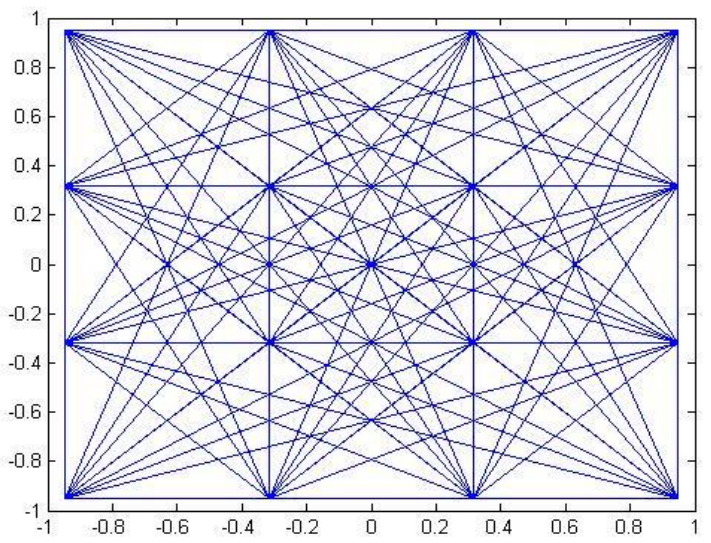

Figure 6: Modulated OFDM data symbols

The equispaced pilot symbols and data are now constructed in the OFDM frame at even and odd positions. Pilot symbols can be viewed as shown in figure 7 .

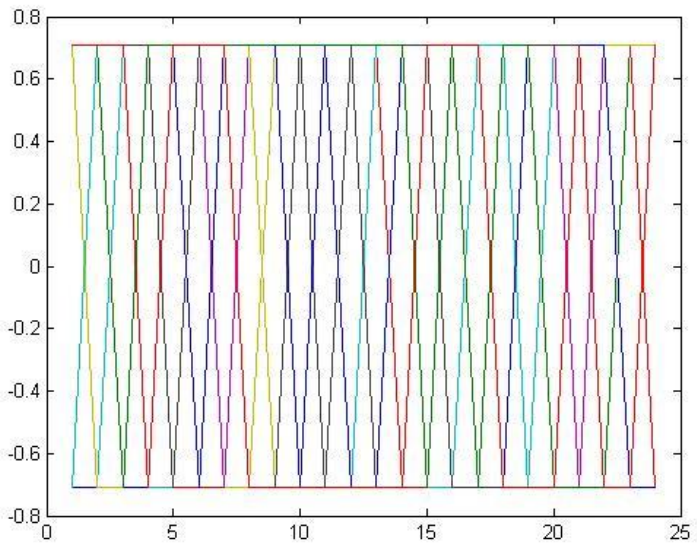

Figure 7: Pilot symbols representation

And data symbols can be viewed as,

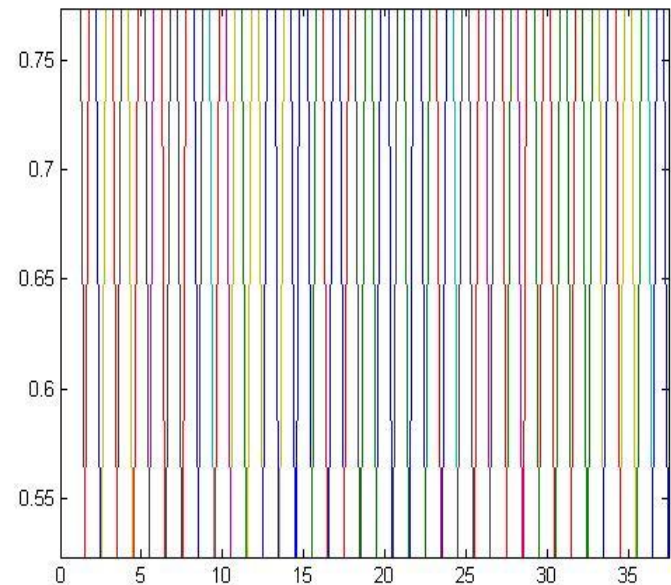

Figure 8. Data symbols representation
Both Pilot symbols and data symbols constructed in OFDM frame is shown in figure 9:

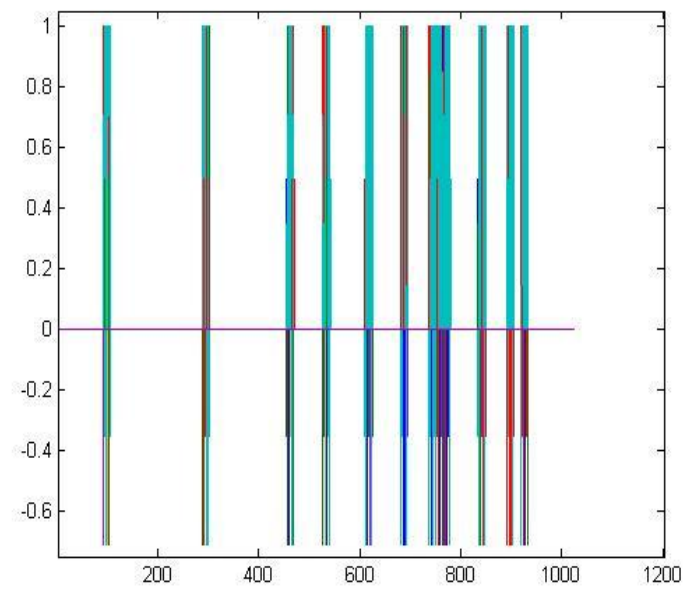

Figure 9: Data and pilot representation in OFDM frame

The formation of OFDM frame can be seen as shown in figure 10 :

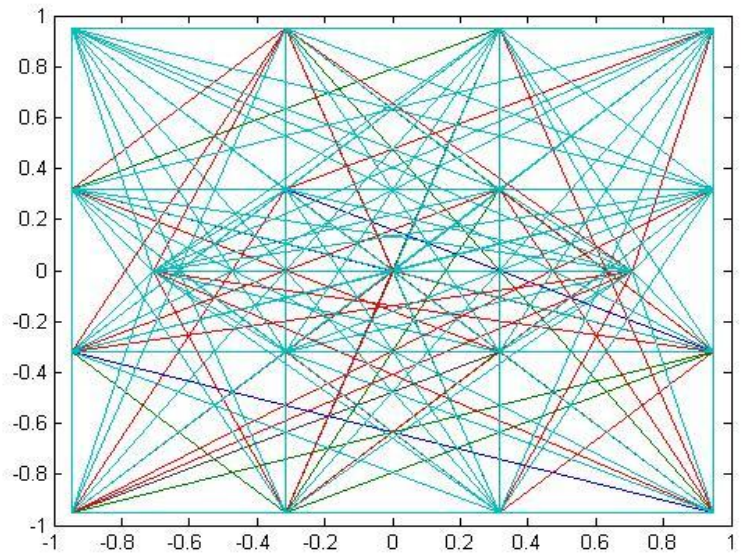

Figure 10: Formation of OFDM frame

The next process in OFDM signal generation is taking the inverse fast fourier transform which converts the data in time domain. After that Cyclic Prefix is inserted which is responsible to avoid inter symbol interference. $\mathrm{CP}=1 / 8$ is taken in the simulation process. In this paper 128 bits of cyclic prefix is inserted in OFDM frame..Finally we do reshaping of resultant data.

The data is received after passing through Rayleigh channel model and additive white Gaussian noise is added to the channel. At the receiver side we proposed different channel estimation whose performance comparison can be shown in Table 2.Among all channel estimation techniques LMS proves its efficiency as compared to No channel estimation, LS (Least Square) and LMMSE (Linear Minimum Mean Square Error) as shown in the following results from figure 11 to figure 17. 
Table 2: BER Vs SNR for Different Channel Estimation Techniques

\begin{tabular}{|c|c|c|c|c|c|c|c|}
\hline \multirow[t]{2}{*}{ SNR } & \multirow{2}{*}{$\begin{array}{c}\text { No } \\
\text { channel }\end{array}$} & \multirow[t]{2}{*}{ LS } & \multirow[t]{2}{*}{ MMSE } & \multicolumn{4}{|c|}{ BER(Bit Error Rate) } \\
\hline & & & & $\operatorname{Itr}=1$ & $\mathrm{Itr}=2$ & $\mathrm{Itr}=3$ & $\mathrm{Itr}=4$ \\
\hline 1 & 0.2255 & 0.1775 & 0.1935 & 0.1828 & 0.1779 & 0.17 & 0.16 \\
\hline 2 & 0.2216 & 0.1624 & 0.1827 & 0.1668 & 0.162 & 0.1673 & 0.155 \\
\hline 3 & 0.2193 & 0.1549 & 0.1765 & 0.1435 & 0.143 & 0.142 & 0.132 \\
\hline 4 & 0.2096 & 0.1387 & 0.1665 & 0.1335 & 0.133 & 0.132 & 0.122 \\
\hline 5 & 0.2053 & 0.1368 & 0.1629 & 0.1215 & 0.121 & 0.12 & 0.11 \\
\hline 6 & 0.2044 & 0.1272 & 0.1617 & 0.1169 & 0.116 & 0.106 & 0.096 \\
\hline 7 & 0.2031 & 0.1199 & 0.1563 & 0.1089 & 0.108 & 0.103 & 0.083 \\
\hline 8 & 0.1994 & 0.1130 & 0.1509 & 0.1081 & 0.1071 & 0.101 & 0.071 \\
\hline 9 & 0.1995 & 0.1099 & 0.1497 & 0.1008 & 0.103 & 0.1001 & 0.07 \\
\hline 10 & 0.1968 & 0.1064 & 0.1476 & 0.0998 & 0.0898 & 0.088 & 0.068 \\
\hline 11 & 0.1973 & 0.1063 & 0.1465 & 0.0991 & 0.0891 & 0.0871 & 0.0571 \\
\hline 12 & 0.1945 & 0.1026 & 0.1426 & 0.0989 & 0.0859 & 0.0759 & 0.0545 \\
\hline 13 & 0.1944 & 0.1007 & 0.1431 & 0.0893 & 0.0763 & 0.0653 & 0.0498 \\
\hline 14 & 0.1926 & 0.1001 & 0.1393 & 0.0859 & 0.0739 & 0.0635 & 0.0455 \\
\hline 15 & 0.1944 & 0.0998 & 0.1413 & 0.0845 & 0.0725 & 0.0629 & 0.0429 \\
\hline
\end{tabular}

Table 2. shows the performance of different channel estimation methods between Bit Error Rate and Signal to Noise ratio.Table shows LMS iterative algorithm upto 4 iterations.

Following graphs shows the peformance of different channel estimation algorithms and which shows that LMS iterative algorithm performs better results than LS and LMMSE algorithms.

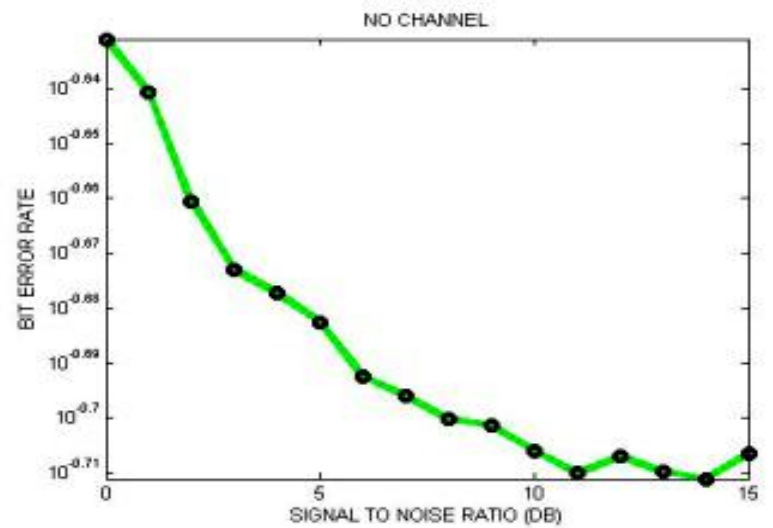

Figure 11: BER V/s SNR Graph for NO Channel Estimation

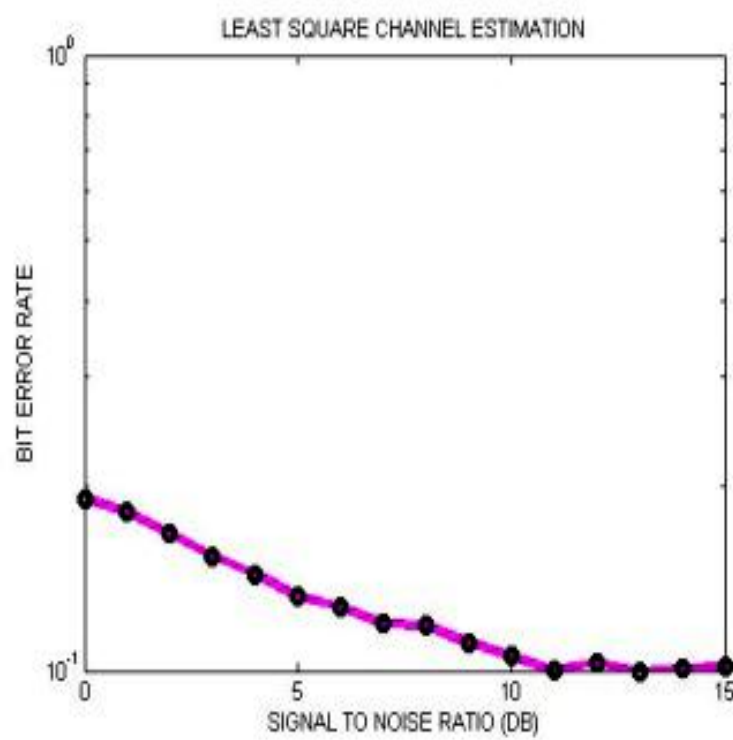

Figure 12: BER v/s SNR graph for Least Square 


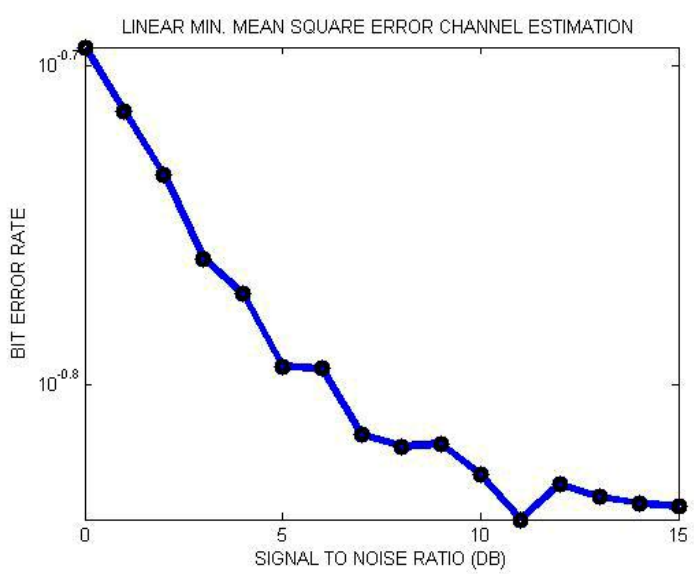

Figure13: BER v/s SNR graph for LMMSE

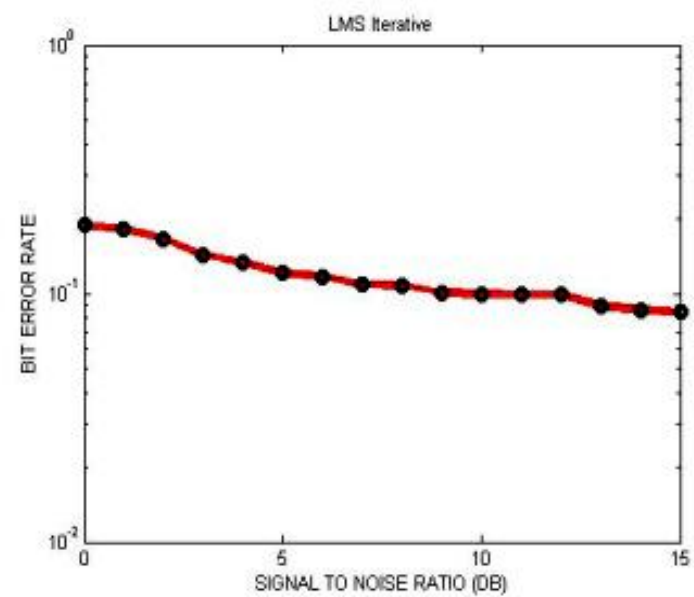

Figure14: BER v/s SNR graph for LMS Iterative with Itr $=1$

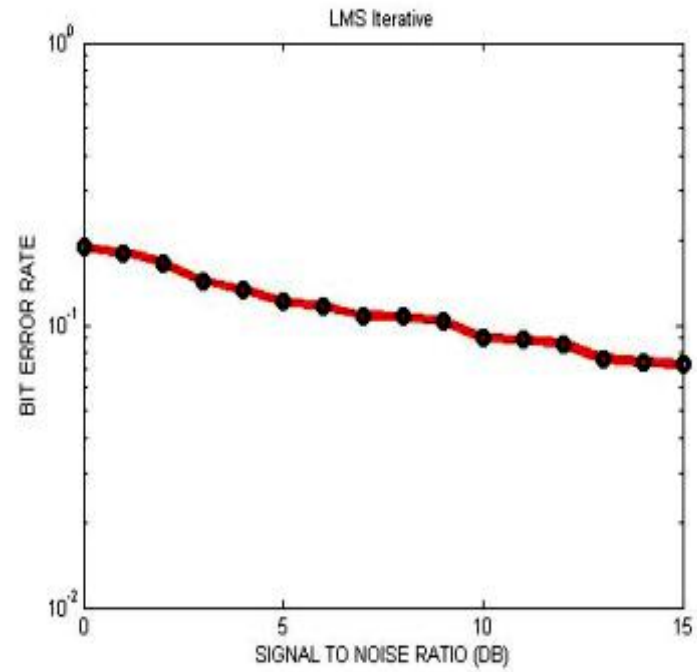

Figure 15: BER v/s SNR graph for LMS Iterative with Itr $=2$

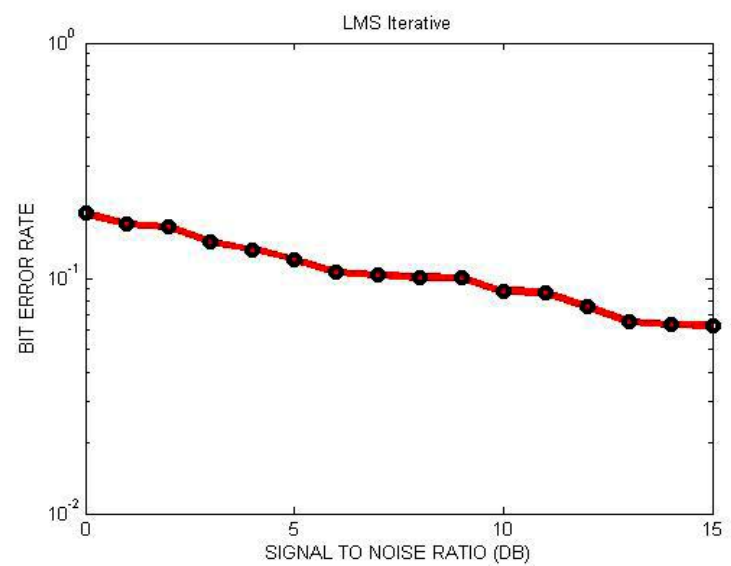

Figure 16: BER v/s SNR graph for LMS Iterative with Itr $=3$

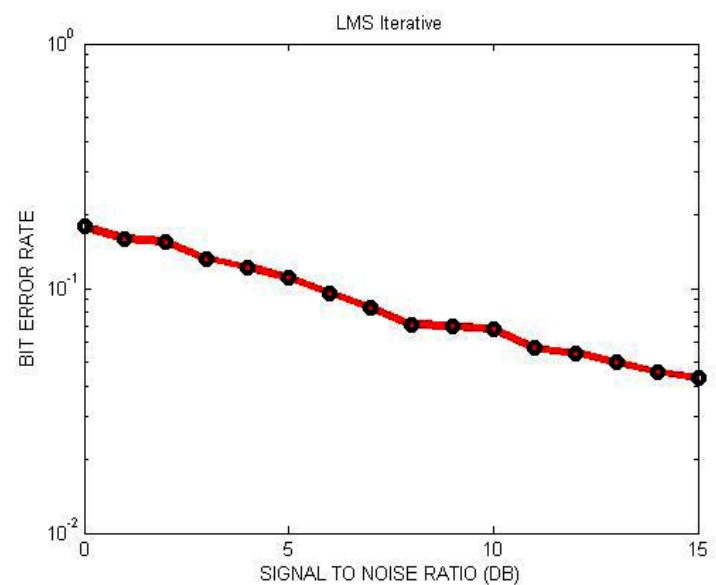

Figure17: BER v/s SNR graph for LMS Iterative with Itr $=4$

\section{CONCLUSION}

In this paper we proposed the performance of different channel estimation techniques. The estimators in this study can be used to efficiently estimate the channel in an OFDM system given a certain knowledge about the channel statistics. The MMSE estimator assumes a priori knowledge of noise variance and channel covariance. Moreover, its complexity is large compared to the LS estimator. For high SNRs the LS estimator is both simple and adequate. However, for low SNRs, the presented modifications of the MMSE and LS estimators will allow a compromise between estimator complexity and performance. To improve the performance of receiver structure an efficient LMS iterative algorithm is added with the receiver, which includes the side information in each iteration as a feedback to the system to improve the BER performance of the system close to the ideal channel performance.LMS algorithm has a higher efficiency than conventional methods and it can work in lower amount of SNR. 


\section{REFERENCES}

[1] IEEE Part II: Wireless LAN Medium Access Control (MAC) and Physical Layer (PHY) Specifications: High-Speed Physical Layer in $5 \mathrm{GHz}$ Band. IEEE Sts. 802.11a-1999, 1999.

[2] J. S. Chow, J. C. Tu, and J. M. Cioffi.A discrete multitone transceiver system for HDSL application.IEEE J. Select. AreasCommun., 9:895908, Aug. 1991.

[3] Z. Wang and G. B. Giannakis.Wireless multicarrier communications where Fourier meets Shannon.IEEE Signal ProcessingMagazine, pages 29-48, May 2000.

[4] P. Hoeher, S. Kaiser and I. Robertson, "Two di mensional pilot-symbol-aided channel estimation by WienerFiltering, "inProc.IEEEICASSP'97, pp.18451848, April 1997.

[5] Coleri, S., Ergen, M., Puri, A., and Bahai, A., "Channel Estimation Techniques Based on Pilot Arrangement in OFDM Systems, " IEEE Transactions on Broadcasting, vol. 48, pp. 223-229, Sept. 2002.

[6] Y e. Li, "Pilot-symbol-aided channel estimation for OFDM in wireless systems," IEEE Trans. Veh. Technol., vol. 49, no. 4, pp. 1207-1215, 2000

[7] Ottersten, B., Viberg, M., and Kailath, T. "Performance Analysis of the Total Least Squares ESPRIT Algorithm," IEEE Transactions on Signal Processing, vol. 39, pp. 1122-1135, May 1991.

[8] Hou, X., Li, S., Liu, D., Yin, C., and Yue, G., "On Two-dimensional Adaptive Channel Estimation in OFDM Systems," 60th IOEEE Vehicular Technology Conference, Los Angeles, Ca., vol. 1, pp. 498-502, Sept. 2004.

[9] Sanzi, F., Sven, J., and Speidel, J., "A Comparative Study of Iterative Channel Estimators for Mobile OFDM Systems," IEEE Transactions on Wireless Communications, vol.2, pp. 849-859, Sept. 2003.

[10] Li, Y., "Simplified Channel Estimation for OFDM Systems with Multiple Transmit Antennas," IEEE Transactions on Communications, vol. 1, pp. 67-75, January 2002.
[11] J. J. van de Beek, O. Edfors, M. Sandell, S. K. Wilson, and P.O. Börjesson, "On channel estimation in OFDM systems," in Proc. IEEE Vehicular Technology Conf., vol. 2, Chicago, IL, July 1995, pp. 815-819.

[12] Coleri, S., Ergen, M., Puri, A., and Bahai, A., "Channel Estimation Techniques Based on Pilot Arrangement in OFDM Systems, " IEEE Transactions on Broadcasting, vol. 48, pp. 223-229, Sept. 2002.

[13] Louis L. Scharf, Statistical Signal Processzng, Addison-Wesley, 1991

[14] Chen, J., C. Chiang and L. Lo, 2007. Iterative Channel Estimation for MIMO OFDM Systems. Proceeding of the International Symposium on Intelligent Signal Processing and Communication Systems, Nov. 28-Dec. 1, IEEE Xplore Press, Xiamen, $\quad$ pp: 774-777. DOI: 10.1109/ISPACS.2007.4446002.

[15] Qiao, Y., S. Yu, P. Su and L. Zhang, 2005. Research on an iterative algorithm of $L S$ channel estimation in MIMO OFDM systems. IEEE Trans. Broadcast.,51: 149-153. DOI: 10.1109/TBC.2004.842524

\section{AUTHOR'S PROFILE}

Rajeshwar singhis M.Tech. student in the Department of Electronics and communication at Lovely Professional University Phagwara,Punjab.He received his B.Tech. from same University in September 2011.He has worked as an engineer in Airtel Telecom for 6 months. His research interests include communication theory and Adhocnetworks.

Mr.Gurpreet Singh Sainireceived his M.Tech. degree from Lovely Professional University in 2012 and worked as an engineer in AIRTEL TELECOM 6 months.. Since January 2012, he has been with Lovely Professional University,Phagwara, as a Asst.Professor. His research interests lie in the area of wireless communication. He has published one national and one international journal in Wimax technology. He has interest in enhancing Mobile Wimax technology with channel estimation algorithms and protocols of Mobile Ad-hoc networks. 